\title{
Experiência Farmácia Solidária: acesso a medicamentos, cidadania, proteção ambiental
} e economicidade

\author{
Farmácia Solidária Experience: access to medication, citizenship, environmental \\ protection and economy
}

\section{Experiencia Farmácia Solidária: acceso a medicamentos, ciudadanía, protección ambiental y economicidad}

Recebido: 05/11/2019

Aprovado: 25/06/2020

Publicado: 18/04/2021

\section{Rodrigo Rodrigues Silva ${ }^{1}$ Ana Carolina Parolini Borges Durante ${ }^{2}$ Gabriela Terra Silva ${ }^{3}$ Luci Mara da Silva ${ }^{4}$ Cecílio de Souza Araújo Filho 5 Claudio Galeno Caldeira 6}

Este é um relato de experiência que tem como objetivo descrever a iniciativa Farmácia Solidária, voltada à captação e redistribuição de medicamentos doados e seus impactos. A iniciativa foi inaugurada no dia 30 de agosto de 2017 e aqui se apresenta dados até agosto de 2018. Os seguintes itens são destacados: Acesso a medicamentos, Economicidade, Proteção ambiental e menor desperdício de medicamentos e Modelo estadual de inovação. Dos medicamentos captados, 170.788 unidades (155.222 medicamentos não controlados; 15.566 medicamentos sujeitos a controle especial) foram repassadas à população, em um total de 3.995 atendimentos; impediu-se que 15.721 .222 unidades de medicamentos se tornassem lixo ambiental e foram promovidas economias da ordem de $\mathrm{R} \$ 311.549,01$ para a Administração Pública e de $\mathrm{R} \$ 389.436,00$ para a população. Constatou-se significativa expansão na oferta de medicamentos não padronizados pelo Sistema Único de Saúde, economicidade indireta (administração pública) e direta (população), impacto ambiental positivo com diminuição de medicamentos vencidos nos domicílios e passíveis de descarte indevido no meio ambiente e, ainda, fluxo direto para as Instituições de Longa Permanência para Idosos. A acreditação de municípios interessados nessa iniciativa encontra-se em via de implantação, com a perspectiva da criação de uma rede intermunicipal mineira.

Descritores: Acesso a medicamentos essenciais e tecnologias em saúde; Meio ambiente; Participação da comunidade; Economia.

This is an experience report that aims to describe the Farmácia Solidária initiative, aimed at capturing and redistributing donated medications, and their impacts. The initiative was initiated on August 30, 2017 and data is presented until August 2018. The following items are highlighted: Access to medication, Economics, Environmental protection and less waste of medicines and State model of innovation. Of the drugs collected, 170,788 units (155,222 non-prescription drugs; 15,566 prescription drugs) were passed on to the population, in a total of 3,995 services; prevented 15,721,222 medication units from becoming environmental waste and promoted savings in the order of $\mathrm{R} \$ 311,549.01$ for the Public Administration and $\mathrm{R} \$$ $389,436.00$ for the population. There was a significant expansion in the supply of medicines not standardized by the Unified Health System, indirect (public administration) and direct (population) economics, positive environmental impact with a decrease in expired medicines in domestic households and subject to improper disposal in the environment, and, direct flow to Long Term Care Institutions for the Elderly. The accreditation of municipalities interested in this initiative is being implemented, with the perspective of creating an intermunicipal mining network.

Descriptors: Access to essential medicines and health technologies; Environment; Community participation; Economics.

Este es un informe de experiencia que pretende describir la iniciativa Farmácia Solidária, centrada en la recogida y redistribución de medicamentos donados y sus impactos. La iniciativa se inauguró el 30 de agosto de 2017 y aquí se presentan los datos hasta agosto de 2018. Se destacan los siguientes puntos: Acceso a los medicamentos, Economicidad, Protección ambiental y menor desperdicio de medicamentos y Modelo estatal de innovación. De los medicamentos recogidos, 170.788 unidades (155.222 medicamentos no controlados; 15.566 medicamentos sometidos a control especial) pasaron a la población, en un total de 3.995 atenciones; se evitó que 15.721.222 unidades de medicamentos se convirtieran en residuos ambientales y hubo un ahorro del orden de $\mathrm{R} \$ 311.549,01$ para la Administración Pública y $\mathrm{R} \$ 389.436,00$ para la población. Hubo una expansión significativa en el suministro de medicamentos no estándar por parte del Sistema Único de Salud, economicidad indirecta (administración pública) y directa (población), impacto ambiental positivo con la reducción de medicamentos caducados en los hogares y susceptibles de ser desechados de forma inadecuada en el medio ambiente y, también, flujo directo a las Instituciones de Larga Estancia para Ancianos. La acreditación de los municipios interesados en esta iniciativa está en marcha, con la perspectiva de crear una red intermunicipal en Minas Gerais.

Descriptores: Acceso a medicamentos esenciales y tecnologías sanitarias; Ambiente; Participación de la comunidad; Economía.

1. Farmacêutico. Mestre em Assistência Farmacêutica. Farmacêutico do Departamento de Assistência Farmacêutica da Prefeitura Municipal de Uberaba (PMU). Doutorando em Atenção à Saúde da Universidade Federal do Triângulo Mineiro. Uberaba, MG, Brasil. ORCID: 0000-0003-2578-4756 E-mail: rodriguesrrs@hotmail.com

2. Farmacêutica. Especialista em Gestão da Assistência Farmacêutica. Farmacêutica do Departamento de Assistência Farmacêutica da PMU. Uberaba, MG, Brasil. ORCID: 0000-0003-0450-2158 E-mail: carolpbdurante@gmail.com

3. Farmacêutica. Especialista em Saúde Pública e da Família. Farmacêutica da Secretaria de Saúde da PMU. Uberaba, MG, Brasil. ORCID: 0000-0002-9843-2121 E-mail: gabbyy.ts@bol.com.br

4. Farmacêutica Bioquímica. Mestre em Ciências da Saúde. Farmacêutica da Secretaria de Saúde da PMU. Uberaba, MG, Brasil. 0RCID: 0000-0002-6673-9786 E-mail: luci-mara@bol.com.br

5. Advogado. Especialista em Direito da Administração Pública. Auditor em Direito do SUS pela PMU. Uberaba, MG, Brasil. ORCID: 0000-0001-5792-8246 E-mail: ceciliofilho@hotmail.com

6. Farmacêutico Bioquímico. Mestre em Educação. Farmacêutico da Secretaria de Saúde da PMU. Uberaba, MG, Brasil. ORCID: 0000-0003-1410-4559 E-mail: cgaleno68@gmail.com 


\section{INTRODUÇÃO}

A

s análises de consumo de medicamentos comprovam que parte importante da renda dos brasileiros destina-se à aquisição de medicamentos, não tratando-se de um desafio recente. Dados da Pesquisa de Orçamentos Familiares (POF) constataram que partir da análise dos dados dos inquéritos realizados em 2002-2003 e 2008-2009, e que após descontados os gastos com saúde da renda das famílias houve acréscimo em 2,6\% e 2,3\%, respectivamente, do número de domicílios vivendo abaixo da linha da pobreza estabelecida pelo Banco Mundial, sendo que o gasto com medicamentos contribuiu com 60,9\% e 65,7\% para este aumento ${ }^{1}$.

Estudos apontam associações entre o acesso a medicamentos e fatores sociodemográficos ${ }^{2,3}$ e, condições gerais de saúde ${ }^{4,5}$. Diferenças socioeconômicas relacionadas ao acesso também são observadas de forma heterogênea em níveis regionais, sobretudo nas grandes regiões brasileiras. Maiores prevalências de acesso a medicamentos são observadas na região Sul, e as menores, nas regiões Norte e Nordeste ${ }^{2-6}$.

Em 2016, apesar da diminuição do orçamento da Saúde, em decorrência das medidas de austeridade, os recursos federais destinados a medicamentos aumentaram 19,6\% em termos reais, chegando à marca de 15,8\% dos recursos do Ministério da Saúde7.

Entre 2008 a 2015, o Orçamento Federal do Acesso a Medicamentos no Brasil (OTMED) aumentou 64,9\% em termos reais, uma elevação muito superior à observada no orçamento da Saúde, de 36,7\% no mesmo período ${ }^{7}$. Assim, a participação percentual do OTMED no orçamento do Ministério da Saúde, que passou de 11,6\% para 14,6\% no mesmo período, se aproximava da média calculada para os países de renda média-alta, que é da ordem de $15 \% 7$.

0 setor de medicamentos possui outro desafio que, embora mais velado, oferece riscos ambientais e à saúde das populações com grandes proporções: o descarte doméstico incorreto de medicamentos. Segundo o Instituto Nacional de Informações Tóxico-Farmacológicas (SINITOX) ${ }^{8}$, o descarte domiciliar de medicamentos na rede de esgoto (vasos sanitários e ralos de pias), faz com que uma parte desse medicamento, após passar pela estação de tratamento de esgoto, contamine o lençol freático e em seguida os rios e oceanos.

0 mesmo destino ocorre com os medicamentos que são jogados no lixo residencial, que após irem para aterros comuns, podem contaminar o solo e rios. Em seguida, por ser um processo cíclico, as pessoas consomem a água desses rios ou os alimentos que com ela entram em contato, expondo sua saúde a riscos. Outro perigo originado com o descarte de medicamentos no lixo doméstico é o risco deles serem reutilizados por catadores de lixo, adultos ou crianças, podendo causar intoxicações ${ }^{8}$. Assim, o objetivo do presente estudo é descrever a iniciativa Farmácia Solidária, voltada à captação e redistribuição de medicamentos doados, e seus impactos.

\section{MÉTODO}

0 estudo caracteriza-se como um relato de experiência, de uma iniciativa da Prefeitura Municipal de Uberaba, representada pela Secretaria Municipal de Saúde e Departamento de Assistência Farmacêutica, denominada Farmácia Solidária, farmácia pública voltada à captação e redistribuição de medicamentos doados.

O município de Uberaba, sede da presente iniciativa, está localizado a $481 \mathrm{~km}$ a oeste da capital estadual, com população estimada em 333.783 habitantes ${ }^{9}$ e dividido em três distritos sanitários (DS). Trata-se de município polo da região ampliada de saúde Triângulo Sul em Alta Complexidade, sede da Superintendência Regional de Saúde (SRS), também referência para a microrregião de Uberaba em Média Complexidade.

Implantada no dia 30 de agosto de 2017, a Farmácia Solidária (DS II), além de possuir uma farmácia básica, possui uma segunda vertente, a farmácia de medicamentos doados. Esses medicamentos são ofertados pela comunidade, instituições e profissionais de saúde, 
instituições filantrópicas, entre outros. Desta forma, a Relação Municipal de Medicamentos Essenciais (REMUME) do município, composta por 136 itens, passou a ser ofertada nesta Farmácia, somada a todas as doações recebidas.

0 presente relato de experiência apresenta dados, especificamente, relativos aos medicamentos doados, aqueles não padronizados/fornecidos pelo Sistema Único de Saúde (SUS). Os medicamentos da vertente farmácia básica (medicamentos padronizados) não foram incluídos.

Para a obtenção dos dados, após a conclusão do $1^{\mathrm{o}}$ ano de atividade, foi conduzida a análise dos dados assistenciais e financeiros relativos ao período de 01/09/2017 a 30/08/2018. Foram contabilizados o número de unidades de medicamentos dispensadas, discriminadas por princípio ativo e apresentações farmacêuticas; a monetização dos medicamentos ofertados à população foi conduzida com base na lista da Câmara de Regulação do Mercado de Medicamentos (CMED), da Agência Nacional de Vigilância Sanitária (ANVISA), a qual estabelece limites de valores financeiros para a compra pública de medicamentos.

\section{RESULTADOS}

A considerar os alcances desta experiência apresentar-se-ão os seguintes itens: Acesso a medicamentos, Economicidade, Proteção ambiental e menor desperdício de medicamentos, e Modelo estadual de inovação.

\section{Acesso a medicamentos}

Em seu $1^{\mathrm{o}}$ ano de funcionamento, o Farmácia Solidária foi responsável por 18.879 atendimentos, dos quais 3.995 envolveram medicamentos oriundos de doações, das quais foram ofertadas 170.788 unidades de medicamentos, majoritariamente, não contemplados em nenhuma Relação de Medicamentos Essenciais do SUS. As unidades dispensadas nestes atendimentos estão demonstrados nas Figuras 1 e 2.

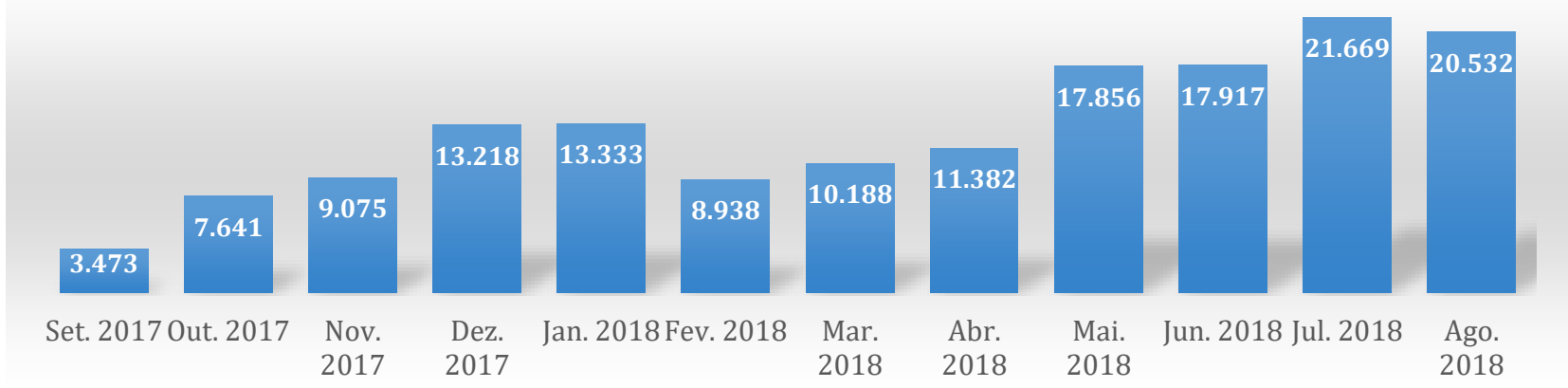

Figura 1. Medicamentos (unidades dispensadas) não sujeitos a controle especial repassados à população pelo Farmácia Solidária. Uberaba, 2018.

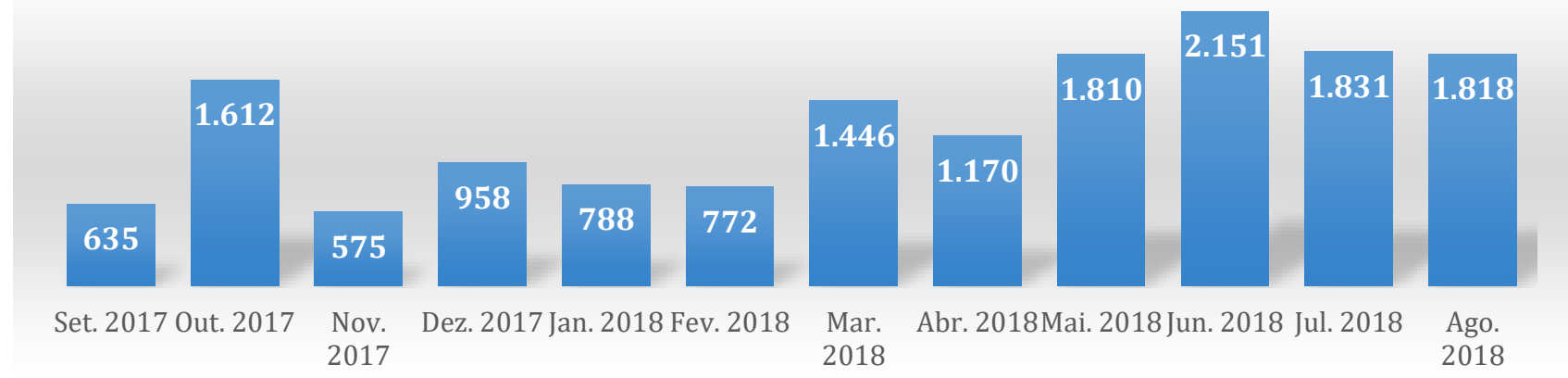

Figura 2. Medicamentos (unidades dispensadas) sujeitos a controle especial repassados à população pelo Farmácia Solidária. Uberaba, MG, 2018. 


\section{Economicidade}

Para mensurar o impacto econômico da Farmácia Solidária em relação à população e à administração pública de Uberaba, todos os medicamentos ofertados foram relacionados e tiveram seus valores (em R\$) levantados, com destaque para:

- a administração pública, especificamente, a Secretaria Municipal de Saúde de Uberaba, alcançou a economia indireta de $\mathrm{R} \$ 311.549,01$, sendo $\mathrm{R} \$ 277.992,61$ em medicamentos não sujeitos a controle especial, e $\mathrm{R} \$ 33.556,40 \mathrm{com}$ medicamentos sujeitos a controle especial (Portaria 344/98). Essa economia indireta pode ser explicada pelo fato de que medicamentos não padronizados pelo SUS, muitas vezes, são demandados via judicialização, o que concorre a custos elevados e imprevisíveis à gestão pública em saúde;

- a população do município beneficiou-se de uma economia direta na compra de medicamentos da ordem de $\mathrm{R} \$ 389.436,00$.

\section{Proteção ambiental e menor desperdício de medicamentos}

Todos os medicamentos repassados para a população pela Farmácia Solidária alcançaram usuários do SUS e se converteram em terapias medicamentosas, ou seguiram o descarte correto de resíduos.

Considerando todos os medicamentos captados, repassados ou não à população, podese afirmar que em seu $1^{\circ}$ ano de atividades, que esta iniciativa evitou que 15.721.222 unidades (de medicamentos), na maioria comprimidos, e em menor parte soluções e suspensões, alcançassem o meio ambiente de forma indevida.

\section{Incentivo à cidadania e participação social}

Com o intuito de fomentar a solidariedade e impactar ainda mais sobre a assistência à saúde no município, a administração pública criou um subprojeto no qual, periodicamente, medicamentos diversos e bem selecionados eram direcionados a nove Instituições de Longa Permanência para Idosos (ILPI) da cidade. Assim, a iniciativa Farmácia Solidária ultrapassou conquistas financeiras e assistenciais, tendo gerado impactos superestruturais de complexa mensuração.

\section{Modelo estadual de inovação}

Após alguns meses de atividade da Farmácia Solidária, convites de consultoria e apoio à multiplicação dessa iniciativa começaram a surgir. Por conta da visibilidade alcançada ao longo de seus primeiros meses, em junho de 2018, representantes do Departamento de Assistência Farmacêutica, da Secretaria Estadual de Saúde (SES) de Minas Gerais, visitaram a iniciativa em busca de mais informações e da melhor maneira de multiplicar a proposta pelo Estado.

\section{DISCUSSÃO}

Considerando que a REMUME do município possui 136 tipos de medicamentos e a Farmácia Solidária ofertou mais 650 novos tipos de medicamentos, pode-se afirmar que essa iniciativa gerou expansão indireta de 4,8 vezes na oferta pública municipal de medicamentos. Além disso, devido ao alto percentual de medicamentos não padronizados pelo SUS, e ofertados gratuitamente, constatou-se impacto direto sobre os gastos da população com medicamentos. Esses medicamentos não padronizados só podem ser obtidos por meio de compras diretas em drogarias, ou por meio de processos de judicialização em desfavor de uma ou mais esferas da gestão pública.

Com base em inferência, cada um dos 3.995 atendimentos gerou a economia direta de R\$ 97,48 para cada usuário. Considerando o impacto dos gastos em saúde dos brasileiros, a ação dessa iniciativa sobre a renda da população do município apresenta-se de forma clara e consistente, diminuindo o impacto de um dos maiores 'vilões' do orçamento familiar: "a compra de medicamentos".

Um estudo nacional ${ }^{2}$ evidencia que o maior número de morbidades e o pior estado de saúde propiciam o menor o número de medicamentos obtidos com gratuidade, quando 
comparados a indivíduos com perfis diferentes. Nesse sentido, em decorrência da diversidade dos produtos ofertados e do baixo grau de burocracia, a presente iniciativa conseguiu alterar essa lógica.

Apesar de implementar a Política Nacional de Resíduos Sólidos (PNRS) ${ }^{10}$, o Brasil convive com o desafio de reverter as toneladas de medicamentos que vencem nas residências do país em iniciativas de aproveitamento, ou pelo menos, descarte correto, sem prejuízos ambientais.

A Farmácia Solidária tem evitado que número elevado e crescente de medicamentos expirem suas validades nas residências sem um destino útil. Todos os medicamentos recebidos sob a forma de doações foram destinados a um tratamento terapêutico apropriado ou, minimamente, foram incluídos em um ciclo correto de descarte de resíduos.

A construção da saúde não pode ser encarada com unilateralidade, projetando sobre os órgãos governamentais todas as expectativas de reorientação dos modelos assistenciais e superação de desafios. Por esse motivo, propostas importantes encorajam a formação de cidadãos cada vez mais envolvidos em projetos sociais e conscientes dos processos que colaboram com a saúde individual e coletiva.

Uma dessas propostas, reconhecida mundialmente, é a 'Carta de Ottawa' (Canadá, 1986) ${ }^{11}$, que impulsionou o movimento de Promoção da Saúde, o qual pode ser definido como:

"Processo de capacitação da comunidade para atuar na melhoria de sua qualidade de vida e saúde, incluindo uma maior participação no controle deste processo". Enfatiza ainda que: "Para se atingir um estado completo de bem estar físico, mental e social os indivíduos e grupos devem saber identificar aspirações, satisfazer necessidades e modificar favoravelmente o meio ambiente." 11.

Uma análise multifocal da iniciativa Farmácia Solidária, bem como da percepção dos usuários acerca dessa iniciativa, evidencia a ampliação paulatina de consciência social e participação da comunidade. Em diversos pontos da Rede de Atenção à Saúde do município e, principalmente na própria Farmácia, observa-se manifestações de solidariedade, nas quais famílias e vizinhanças se mobilizam na captação de medicamentos nos arredores de suas residências; ou mesmo pessoas que, individualmente, promovem impactos sobre grandes grupos.

As doações oriundas de instituições de saúde, consultórios médicos e organizações filantrópicas também merecem destaque e reforçam o engajamento crescente da sociedade na presente iniciativa.

Um dos pontos de grande relevância, e aventado na discussão com representantes da SES, foi a possibilidade de difundir essa iniciativa para muitos municípios mineiros para, posteriormente, estabelecer parcerias intermunicipais envolvendo permuta de medicamentos doados, com ênfase naqueles não padronizados pelo SUS, ou em desabastecimento em determinados municípios por questões circunstanciais. Essas parcerias podem promover benefícios assistenciais, financeiros, ambientais e sociais em todo o estado, além de otimizar o aproveitamento dos medicamentos devido às particularidades epidemiológicas de cada município.

Em maio de 2019, no 36º Congresso Mineiro de Municípios ${ }^{12}$, organizado pela Associação Mineira de Municípios (AMM), a Farmácia Solidária foi vencedora do Prêmio de Boas Práticas na Gestão Municipal, Eixo Gestão da Saúde (2019), sendo reconhecida como modelo para os demais municípios mineiros no que concerne à expansão do acesso a medicamentos, economicidade para a população e administração pública, proteção ambiental e promoção de cidadania. 


\section{CONCLUSÃo}

O acesso a medicamentos no contexto da promoção, proteção e recuperação da saúde possui grande relevância e está, comumente, ladeado por desafios diversos. Fruto de muito trabalho conjunto, e do apoio de áreas de conhecimento administrativo, farmacêutico, financeiro e assistencial, a Farmácia Solidária traz em sua essência a simplicidade e a robustez de uma iniciativa polivalente.

É relevante mencionar que a Farmácia Solidária enfrenta desafios diários, dentre eles: a insuficiência do quadro atual de recursos humanos, que torna-se aparente em face da demanda de atendimentos; o volume crescente de doações exige a readequação infraestrutural do espaço, para permitir o acondicionamento ideal dos medicamentos; não existe garantia de que as doações recebidas serão repassadas para a população em sua totalidade, o que paulatinamente, tem aumentado o volume de medicamentos encaminhados para o descarte pela farmácia.

No tocante às questões metodológicas, o presente estudo apresenta limitação relacionada à inexistência de dados de demanda reprimida, ou seja, as demandas não atendidas pela Farmácia Solidária, que não constam em nenhuma base de dados (física ou digital) e, por conseguinte, não foram contabilizadas e analisadas.

Apesar das dificuldades apontadas, busca-se encorajar a multiplicação e a criação de uma grande rede de municípios integrantes, e seguirá acreditando que políticas públicas em saúde podem, harmoniosamente, combinar melhorias assistenciais, baixo custo, economias direta e indireta, contribuições ao meio ambiente e à vida em sociedade. Após consultoria, o município de Conceição das Alagoas (MG) foi o primeiro a adotar essa iniciativa, e outros, como Araxá (MG), Guaxupé (MG), Jacutinga (MG) e Barra do Garças (MT) manifestaram interesse e receberam diretrizes iniciais para a implantação.

\section{REFERÊNCIAS}

1. Boing $\mathrm{AC}$, Bertoldi $\mathrm{AD}$, Posenato LG, Peres KG. Influência dos gastos em saúde no empobrecimento de domicílios no Brasil. Rev Saúde Pública [Internet]. 2014 [citado em 21 jun 2020]; 48(5):797-807. DOI: https://doi.org/10.1590/S0034-8910.2014048005113

2. Viana KP, Brito AS, Rodrigues CS, Luiz RR. Acesso a medicamentos de uso contínuo entre idosos, Brasil. Rev Saúde Pública [Internet]. 2015 [citado em 21 jun 2020]; 49:14. DOI: https://doi.org/10.1590/S0034-8910.2015049005352

3. Katrein F, Tejada CAO, Restrepo-Méndez MC, Bertoldi AD. Desigualdade no acesso a medicamentos para doenças crônicas em mulheres brasileiras. Cad Saúde Pública [Internet]. 2015 [citado em 21 jun 2020]; 31(7):1416-26. DOI: https://doi.org/10.1590/0102-311X00083614

4. Bertoldi AD, Barros AJD, Hallal PC, Lima RC. Utilização de medicamentos em adultos: prevalência e determinantes individuais. Rev Saúde Pública [Internet]. 2004 [citado em 21 jun 2020]; 38(2):228-38. DOI: https://doi.org/10.1590/S0034-89102004000200012

5. Vosgerau MZS, Soares DA, Souza RKT, Matsuo T, Carvalho GS. Consumo de medicamentos entre adultos na área de abrangência de uma Unidade de Saúde da Família. Ciênc Saúde Colet. [Internet]. 2011 [citado em 21 jun 2020]; 16(Suppl1):1629-38. DOI: https://doi.org/10.1590/S141381232011000700099

6. Oliveira MA, Luiza VL, Tavares NUL, Mengue SS, Arrais PSD, Farias MR, et al. Acesso a medicamentos para doenças crônicas no Brasil: uma abordagem multidimensional. Rev Saúde Pública [Internet]. 2016 [citado em 21 jun 2020]; 50(Suppl2):6s. DOI: https://doi.org/10.1590/s1518-8787.2016050006161

7. Ministério da Fazenda (Brasil), Secretaria de Finanças. Demonstrativo dos gastos tributários: estimativas bases efetivas - 2013, Série 2011 a 2016 [Internet]. Brasília, DF: Receita Federal do Brasil; 2016 [citado em 21 jun 2020]. Disponível em: https://receita.economia.gov.br/dados/receitadata/renuncia-fiscal/demonstrativos-dos-gastostributarios/arquivos-e-imagens/ano-calendario-2013-serie-2011-a-2016 
8. Sistema Nacional de Informações Tóxico-Farmacológicas (Brasil). Descarte de medicamentos [Internet]. Rio de Janeiro: SINITOX; 2019 [citado em 21 jun 2020]. Disponível em: https://sinitox.icict.fiocruz.br/descarte-de-medicamentos

9. Instituto Brasileiro de Geografia e Estatística, Diretoria de Pesquisas, Coordenação de População e Indicadores Sociais. Estimativas da população residente com data de referência de julho de 2018 [Internet]. Rio de Janeiro: IBGE; 2018 [citado em 21 jun 2020]. Disponível em: https://www.ibge.gov.br/estatisticas/sociais/populacao/9103-estimativas-depopulacao.html?=\&t=o-que-e

10. Presidência da República (Brasil), Casa Civil, Subchefia para Assuntos Jurídicos. Lei no 12.305, de 2 de agosto de 2010. Institui a Política Nacional de Resíduos Sólidos; altera a Lei no 9.605, de 12 de fevereiro de 1998; e dá outras providências [Internet]. Brasília, DF: Casa Civil; 2010 [citado em 21 jun 2020]. Disponível em: http://www2.mma.gov.br/port/conama/legiabre.cfm?codlegi=636 11. Ministério da Saúde (Brasil), Secretaria de Políticas de Saúde, Projeto Promoção da Saúde. Declarações das Conferências de Promoção da Saúde [Internet]. Brasília, DF: MS; 2001 [citado em 21 jun 2020]. Disponível em: https://bvsms.saude.gov.br/bvs/publicacoes/cartas_promocao.pdf 12. 36을 Congresso Mineiro de Municípios; 2019; Belo Horizonte. Belo Horizonte: Associação Mineira de Municípios; 2019.

Editora Associada: Estefânia Maria Soares Pereira

\section{CONTRIBUIÇÕES}

Ana Carolina Parolini Borges Durante, Claudio Galeno Caldeira, Gabriela Terra Silva e Luci Mara da Silva contribuíram na concepção, coleta e análise dos dados. Cecílio de Souza Araújo Filho participou da concepção. Rodrigo Rodrigues Silva atuou na concepção, coleta e análise dos dados, redação e revisão.

\section{Como citar este artigo (Vancouver)}

Silva RR, Durante ACPB, Silva GT, Silva LM, Araújo Filho CS, Caldeira CG. Experiência Farmácia Solidária: acesso a medicamentos, cidadania, proteção ambiental e economicidade. REFACS [Internet]. 2021 [citado em inserir dia, mês e ano de acesso]; 9(2):488-94. Disponível em: inserir link de acesso. DOI: inserir link do DOI

\section{Como citar este artigo (ABNT)}

SILVA, R. R.; DURANTE, A. C. P. B.; SILVA, G. T.; SILVA, L. M.; ARAÚJO FILHO, C. S.; CALDEIRA, C. G. Experiência Farmácia Solidária: acesso a medicamentos, cidadania, proteção ambiental e economicidade. REFACS, Uberaba, MG, v. 9, n. 2, p. 488-94, 2021. DOI: inserir link do DOI. Disponível em: inserir link de acesso. Acesso em: inserir dia, mês e ano de acesso.

\section{Como citar este artigo (APA)}

Silva, R.R., Durante, A.C.P.B., Silva, G.T., Silva, L.M., Araújo Filho, C.S., \& Caldeira, C.G. (2021). Experiência Farmácia Solidária: acesso a medicamentos, cidadania, proteção ambiental e economicidade. REFACS, 9(2), 488-94. Recuperado em inserir dia, mês e ano de acesso de inserir link de acesso. DOI: inserir link do DOI. 